\title{
DEGRADAÇÃO DE FENOL POR PROCESSOS OXIDATIVOS AVANÇADOS
}

\author{
F.S. SOUZA ${ }^{1}$, Y.M. POSSER ${ }^{1}$, L.A.FÉRIS ${ }^{1}$, E. CARISSIMI ${ }^{2}$ \\ ${ }^{1}$ Universidade Federal do Rio Grande do Sul, Departamento de Engenharia Química \\ ${ }^{2}$ Universidade Federal de Santa Maria, Departamento de Engenharia Sanitária e Ambiental \\ E-mail para contato: fesouza@enq.ufrgs.br
}

\begin{abstract}
RESUMO - O fenol é um poluente tóxico encontrado em efluentes industriais e sua remoção é de extrema importância para minimizar os impactos ambientais. Métodos convencionais de tratamento não são eficientes para a completa remoção deste composto. Neste contexto, o objetivo deste trabalho é estudar a degradação do fenol, utilizando Processos Oxidativos Avançados: Fenton $\left(\mathrm{H}_{2} \mathrm{O}_{2} / \mathrm{Fe}^{2+}\right)$, FotoFenton $\left(\mathrm{UV} / \mathrm{H}_{2} \mathrm{O}_{2} / \mathrm{Fe}^{2+}\right)$ e Ozônio $\left(\mathrm{O}_{3}\right)$. Estes processos são baseados na formação de radicais hidroxila, que são altamente reativos e capazes de degradar eficientemente diversos poluentes. Avaliou-se a influência do $\mathrm{pH}$ para o tratamento com Ozônio e a influência da luz UV no reagente Fenton. Através da comparação dos resultados, a maior eficiência de remoção de fenol foi obtida por Ozonização em meio alcalino (95,9\%), seguido por Foto-fenton $(94,9 \%)$. Uma análise comparativa dos tratamentos analisados evidencia o potencial de aplicação destas técnicas em efluentes industriais contendo fenol.
\end{abstract}

\section{INTRODUÇÃO}

O fenol é um composto orgânico aromático de fórmula $\mathrm{C}_{6} \mathrm{H}_{5} \mathrm{OH}$ frequentemente encontrado em efluentes industriais provenientes de indústrias têxteis, da fabricação de resinas, de materiais isolantes, de tintas e corantes, e de produtos farmacêuticos como ácido acetilsalicílico (Aspirina) e o paracetamol. Este poluente caracteriza-se por ser tóxico e persistente no meio ambiente e, mesmo em baixas concentrações, provoca impactos nos recursos hídricos e efeitos nocivos à saúde humana e animal (Zeng et al., 2013).

O fenol consta como contaminante prioritário na lista elaborada pela Agência de Proteção Ambiental (EPA) dos Estados Unidos (Zhong et al., 2012). A Resolução 430/11 do Conselho Nacional do Meio Ambiente (CONAMA) define que os efluentes de qualquer fonte poluidora somente poderão ser lançados, direta ou indiretamente nos corpos de água desde que obedeçam as condições de $0,5 \mathrm{mg} . \mathrm{L}^{-1}$ de fenóis totais. Com a legislação cada vez mais restritiva, é essencial a busca por tecnologias limpas, eficientes e viáveis.

Neste contexto, os Processos Oxidativos Avançados (POAs) apresentam uma alternativa atraente para a completa degradação de poluentes orgânicos como o fenol. Segundo Teixeira e Jardim (2007), os Processos Oxidativos Avançados caracterizam-se por transformar a grande maioria dos contaminantes orgânicos em dióxido de carbono, água e ânions inorgânicos por meio de reações de degradação que envolvem espécies transitórias oxidantes. O principal mecanismo dos POAs é a geração de radicais hidroxila $(\bullet \mathrm{OH})$, que 
reagem de forma rápida e não seletiva com quase todos os compostos orgânicos (Stasinakis, 2008). Dentre os POAs propostos para a remoção de fenóis presentes em matrizes aquosas, águas e efluentes, destacam-se a técnica de ozonização e os processos Fenton e Foto-Fenton (Babuponnusami e Muthukumar, 2012; Souza et al., 2012, Kavitha e Palinivelu, 2003).

A ozonização é um processo eficaz no tratamento devido ao alto potencial de oxidação do ozônio $(2,07 \mathrm{~V})$, possibilitando a completa mineralização do contaminante sem geração de subprodutos, sendo que o tratamento é realizado no local da geração (Souza et al, 2012). Em pH básico, decompõe-se levando à formação de radicais hidroxila cujo potencial de oxidação é ainda mais elevado (2,80 V), conforme Equação 1. Desta maneira, a oxidação de compostos orgânicos durante a ozonização pode ocorrer via ozônio molecular (reação direta predominante em meio ácido) ou radical hidroxila (reação indireta - predominante em meio básico - Processo oxidativo avançado). Em pH neutro, ambos oxidantes podem estar atuando (Jin et al., 2012). Entre os estudos da degradação de fenol com ozônio, destacam os trabalhos de Matheswaran e Moon (2009), Turhan e Uzman (2008).

$$
2 \mathrm{O}_{3}+\mathrm{H}_{2} \mathrm{O} \stackrel{\mathrm{OH}^{-}}{\longrightarrow} \mathrm{HO}^{\bullet}+\mathrm{O}_{2}+\mathrm{HO}_{2}
$$

O processo Fenton consiste na geração de radicais hidroxila através da transferência de elétrons entre o peróxido de hidrogênio e os íons ferrosos (reagentes Fenton) conforme indicado na Equação 2. Os íons de ferro da reação atuam como catalisador e o radical hidroxila é o agente responsável pela degradação dos compostos orgânicos. $\mathrm{O}$ pH do meio reacional é um fator limitante no processo. Segundo Kuo (1992) e Kiwi et al. (2000), em pH próximo a 3, o radical hidroxila é a espécie mais ativa na degradação de compostos orgânicos e em $\mathrm{pH}$ superior a 4 , os íons ferrosos facilmente se convertem a íons férricos que, na presença de hidróxido, produzem complexos capazes de coagular e precipitar a matéria orgânica (Wang et al.,2008). O processo Foto-Fenton é considerado um aprimoramento do Fenton, pois trata-se da aplicação de radiação ultravioleta (UV-A, UV-B e Visível), formando um radical hidroxila adicional através da fotólise dos íons $\mathrm{Fe}^{3+}$ como mostra a Equação 3. Dentre os estudos realizados para tratamento de efluentes contendo fenol através dos processos Fenton e Foto-Fenton, pode-se destacar os trabalhos de Kavitha e Palinivelu (2003), Babuponnusami e Muthukumar (2012) e Karci et al. (2012).

$$
\begin{gathered}
\mathrm{Fe}^{2+}+\mathrm{H}_{2} \mathrm{O}_{2} \rightarrow \mathrm{Fe}^{3+}+\mathrm{HO}^{-}+\mathrm{HO}^{\bullet} \\
\mathrm{Fe}^{3+}+\mathrm{H}_{2} \mathrm{O}+\mathrm{hv} \rightarrow \mathrm{Fe}^{2+}+\mathrm{HO}^{-}+\mathrm{HO}^{\bullet}
\end{gathered}
$$

Visando comparar a eficiência de degradação do fenol, o objetivo geral deste trabalho consiste na aplicação de Processos Oxidativos Avançados: Fenton $\left(\mathrm{H}_{2} \mathrm{O}_{2} / \mathrm{Fe}^{2+}\right)$, Foto-Fenton $\left(\mathrm{UV} / \mathrm{H}_{2} \mathrm{O}_{2} / \mathrm{Fe}^{2+}\right)$ e Ozônio $\left(\mathrm{O}_{3}\right)$. Avaliou-se a influência do $\mathrm{pH}$ para o tratamento com Ozônio e a influência da luz UV no reagente Fenton. De acordo com os resultados encontrados, elaborou-se uma análise comparativa dos tratamentos analisados, evidenciando o potencial de aplicação destas técnicas em efluentes reais contendo fenol, desenvolvendo alternativas de fácil operacionalidade e eficiente para atender às legislações ambientais. 


\section{METODOLOGIA}

A degradação de soluções aquosas de fenol (marca Synth) com concentração inicial de $\left(\mathrm{C}_{0}\right)$ de $100 \mathrm{mg} . \mathrm{L}^{-1}$ foi analisada para os três POAs: ozônio, Fenton e Foto-Fenton. Os experimentos foram realizados em duplicata.

\subsection{Controle analítico}

As medidas de $\mathrm{pH}$ foram determinadas utilizando-se o aparelho pHmetro modelo DM22 da Digimed. A determinação de fenóis totais foi medida pelo método fotométrico direto baseado na reação com a 4-aminoantipirina (APHA, 1995), lido em espectrofotômetro Próanálise, modelo UV-1600 no comprimento de onda de 500nm.

\subsection{Ozonização}

Nos experimentos com ozônio, soluções aquosas de $1,5 \mathrm{~L}$ contendo fenol na concentração de $100 \mathrm{mg} . \mathrm{L}^{-1}$, foram analisadas em $\mathrm{pH} \mathrm{3,} \mathrm{7,} \mathrm{e} \mathrm{10,} \mathrm{ajustadas} \mathrm{pela} \mathrm{adição} \mathrm{de}$ soluções aquosas de $\mathrm{HCl}\left(2 \mathrm{~mol} . \mathrm{L}^{-1}\right)$ e $\mathrm{NaOH}\left(2 \mathrm{~mol} . \mathrm{L}^{-1}\right)$. O sistema de ozonização constitui de: Gerador de Ozônio, coluna de contato de ozônio/líquido com difusor poroso, rotâmetro e cilindro de gás oxigênio, conforme fluxograma apresentado na Figura 1(a). O ozônio foi gerado a partir de oxigênio puro pelo método da descarga elétrica, utilizando-se um ozonizador marca Ozonium Systems. O ozônio foi introduzido na coluna em uma vazão de 1L. $\min ^{-1}$ por meio de um difusor poroso para promover uma eficiente transferência de massa. O ozônio remanescente foi coletado em um frasco lavador com solução de Iodeto de Potássio $2 \%$. Os experimentos de ozonização foram avaliados durante $15 \mathrm{~min}$. Alíquotas de $10 \mathrm{~mL}$ foram coletadas em tempos regulares para a avaliação da degradação do fenol.

\subsection{Fenton e Foto-fenton}

Os experimentos Fenton foram conduzidos à temperatura ambiente em um becker

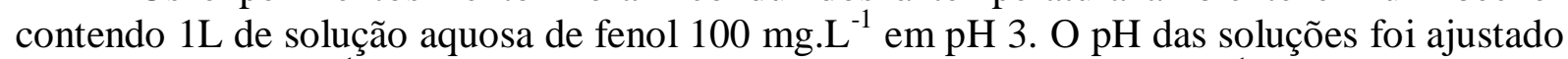
com $\mathrm{HCl}\left(1 \mathrm{~mol} . \mathrm{L}^{-1}\right)$. Após o ajuste do $\mathrm{pH}$, foram adicionados $4 \mathrm{mg} . \mathrm{L}^{-1}$ de sulfato ferroso heptahidratado P.A. (FeSO $4.7 \mathrm{H}_{2} \mathrm{O}$, Synth) e o início dos ensaios ocorreu com a adição de 400 mg. $\mathrm{L}^{-1}$ de peróxido de hidrogênio P.A $\left(\mathrm{H}_{2} \mathrm{O}_{2}\right.$, Synth). As concentrações dos reagentes Fenton foram definidas com base no estudo realizado por Babuponnusami e Muthukumar (2012). A homogeneização do meio reacional nos ensaios Fenton foi realizada através de um agitador magnético (Fisatom modelo 706). Para avaliar a degradação do fenol, foram coletadas alíquotas de $10 \mathrm{~mL}$ de solução em intervalos de tempo de 15 minutos. As amostras coletadas foram ajustadas para $\mathrm{pH} 10$ através da adição de $\mathrm{NaOH}\left(0,5\right.$ mol. $\left.\mathrm{L}^{-1}\right)$ para garantir que a reação Fenton fosse interrompida.

Os experimentos Foto-Fenton foram realizados similarmente aos processos Fenton, porém foram conduzidos em um foto-reator composto por um recipiente de $2 \mathrm{~L}$ (reator) que é inserido em uma câmara (reator com lâmpadas UV) com 4 lâmpadas de radiação ultravioleta de luz negra $8 \mathrm{~W}$ (ECOLUME, fluorescente tipo BLB), totalizando $32 \mathrm{~W}$ de potência. A emissão de radiação de cada lâmpada é de, aproximadamente, $0,8 \mathrm{~mW} / \mathrm{cm}^{2}$ no comprimento de onda de $365 \mathrm{~nm}$. A Figura 1(a) apresenta o fluxograma ilustrativo. O início dos ensaios ocorreu no acionamento das lâmpadas e o tempo total dos ensaios foi de 1hora. A 
homogeneização do meio reacional foi realizada através de um agitador mecânico (Modelo TE-039, Tecnal) e a temperatura foi monitorada através de um termopar (marca Incoterm modelo L-074/04).
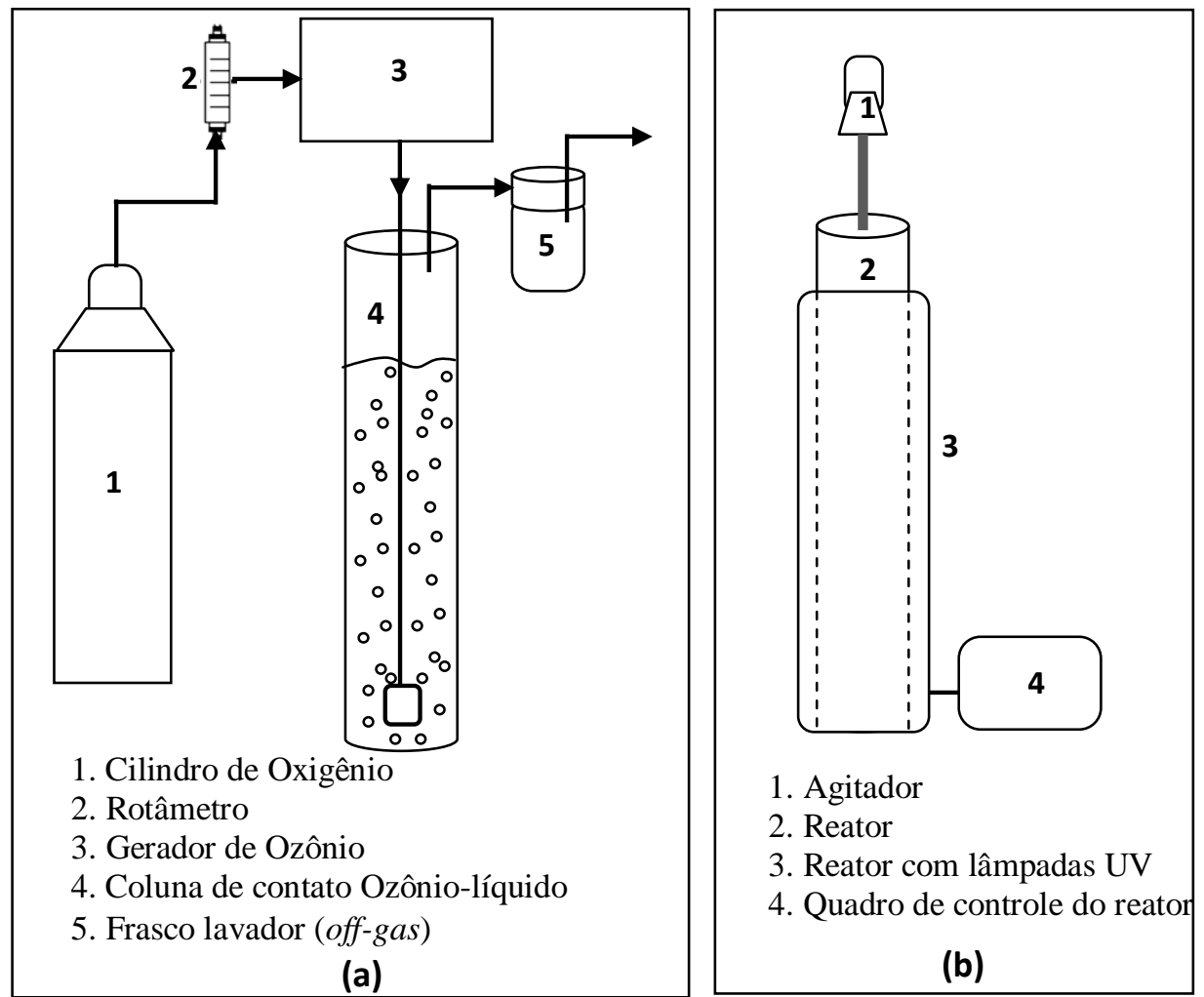

Figura 1 - Fluxograma ilustrativo do: (a) processo de ozonização; (b) foto-fenton.

\section{RESULTADOS E DISCUSSÃO}

A Figura 2 mostra a remoção de fenol pelo processo de ozonização em diferentes tempos de tratamento nos diferentes pHs. Observa-se que os melhores resultados foram obtidos em meio alcalino, alcançando aproximadamente $96 \%$ de eficiência de degradação do fenol, ou seja, a concentração final foi de $4,11 \mathrm{mg} \cdot \mathrm{L}^{-1}$.

Os resultados obtidos comprovam que a eficiência do processo de degradação por ozônio depende do pH da solução. De acordo com Jin et al. (2012), sob condições de pH ácido, ocorre a oxidação direta de compostos orgânicos por ozônio molecular dissolvido. Este mecanismo direto apresenta constantes cinéticas relativamente lentas e geralmente não promove a degradação completa dos compostos orgânicos. Esta limitação pode ser contornada usando soluções alcalinas, onde o ozônio se decompõe e origina radicais hidroxilas $(\bullet \mathrm{OH})$, que reagem rapidamente com a maioria dos compostos orgânicos, aumentando a eficiência do tratamento (Mahmound e Freire, 2007). 




Figura 2 - Ozonização de fenol em soluções aquosas em meio ácido, neutro e alcalino.

$[\text { Fenol }]_{0}=100 \mathrm{mg} . \mathrm{L}^{-1}$.

A Figura 3 apresenta os resultados obtidos para degradação do fenol pelos processos Fenton e Foto-Fenton em intervalos de tempo de 15 minutos, durante os ensaios de 1 hora.

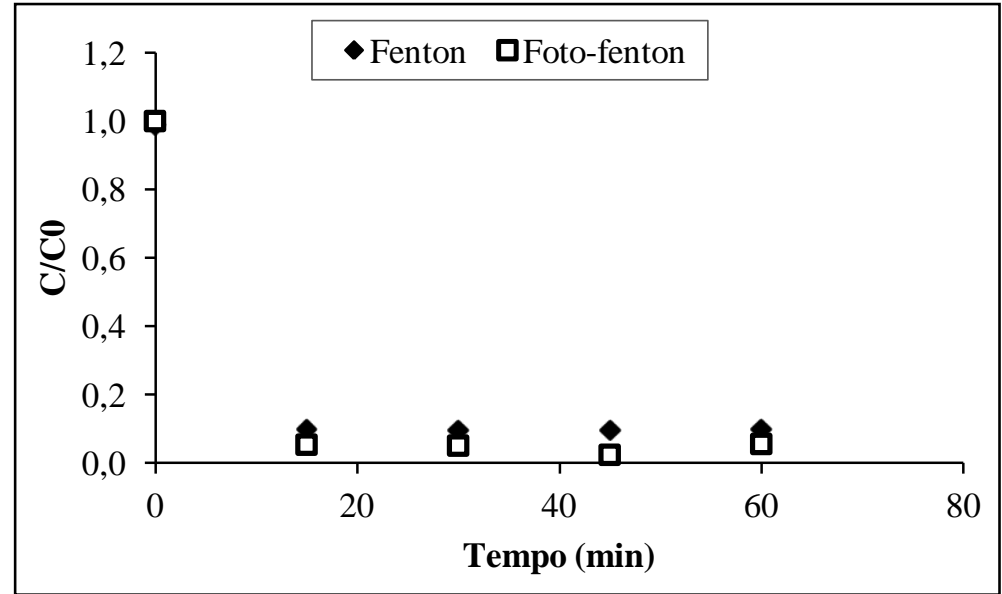

Figura 3 - Processo Fenton e Foto-fenton na degradação de fenol (pH 3, [Fenol $]_{0}=100 \mathrm{mg} . \mathrm{L}^{-1}$ $\left.\left[\mathrm{Fe}^{2+}\right]_{0}=4 \mathrm{mg} \cdot \mathrm{L}^{-1},\left[\mathrm{H}_{2} \mathrm{O}_{2}\right]_{0}=400 \mathrm{mg} \cdot \mathrm{L}^{-1}\right)$.

Os resultados demonstraram que os processos Fenton e Foto-Fenton são capazes de degradar mais de $90 \%$ dos fenóis totais da solução (94,9\% processo Foto-Fenton e 90,3\% processo Fenton) nos minutos iniciais de reação, obtendo concentrações finais de $5,52 \mathrm{mg} . \mathrm{L}^{-1}$ e $10,2 \mathrm{mg} . \mathrm{L}^{-1}$, respectivamente.

Apesar da eficiência dos dois processos serem semelhantes e o processo Fenton gerar menos custos associados pela aplicação de radiação UV, diversos autores indicam que o processo Foto-Fenton apresenta uma vantagem em relação do Fenton na degradação de compostos intermediários gerados durante a reação de degradação do fenol. Os estudos realizados por Karci at. al. (2012) e Kavitha e Palanivelu (2003) evidenciaram que a degradação do fenol através dos processos Fenton e Foto-Fenton é acompanhada pela formação de compostos aromáticos hidroxílicos e clorados, hidroquinona e compostos alifáticos (ácido acético, oxálico e fórmico - ácidos carboxílicos). $\mathrm{Na}$ aplicação do processo Fenton os autores evidenciaram que os compostos alifáticos não foram mineralizados ao final 
da reação e conferiram ao efluente final, após tratamento, uma característica de toxicidade aguda. Já através da aplicação do Processo Foto-Fenton, os estudos realizados indicaram que há a formação de subprodutos nos estágios iniciais de degradação, porém, ao final do ensaio os compostos foram quase totalmente mineralizados. Os autores atribuíram a eficácia do processo Foto-Fenton frente ao processo Fenton à ação da radiação UV.

Analisando comparativamente a eficiência dos processos de ozonização, Fenton e Foto-Fenton na degradação de fenóis em 15 minutos de tratamento, obtiveram-se os resultados apresentados na Figura 4. Observa-se que o processo com Ozônio em meio básico alcançou a melhor eficiência em 15 minutos de tratamento $(95,9 \%)$ quando comparado aos processos Foto-Fenton $(94,9 \%)$, Fenton $(90,3 \%)$ e com ozônio em meio neutro $(73,5 \%)$ e ácido $(60,6 \%)$.



Figura 4 - Eficiências dos ensaios de ozonização ( $\mathrm{pH}$ básico, $\mathrm{pH}$ neutro, $\mathrm{pH}$ ácido) e fenton e foto-fenton $\left(\mathrm{pH} 3,[\mathrm{Fenol}]_{0}=100 \mathrm{mg} . \mathrm{L}^{-1}\left[\mathrm{Fe}^{2+}\right]_{0}=4 \mathrm{mg} . \mathrm{L}^{-1},\left[\mathrm{H}_{2} \mathrm{O}_{2}\right]_{0}=400 \mathrm{mg} . \mathrm{L}^{-1}\right)$ analisados após 15 minutos de ensaio.

\section{CONCLUSÕES}

De acordo com os resultados obtidos, os processos com ozônio em meio alcalino e o processo foto-fenton apresentaram as melhores eficiências de remoção do fenol, sendo 95,9\% e $94,9 \%$, respectivamente, após 15 minutos de tratamento.

$\mathrm{Na}$ aplicação do processo de ozonização foram obtidos resultados satisfatórios em $\mathrm{pH}$ alcalino. Tal técnica apresenta uma vantagem adicional em relação aos processos envolvendo os reagentes Fenton, pois não necessita de insumos para o processo (ferro e peróxido de hidrogênio). Além disso, nos processos Fenton e Foto-Fenton, o pH deve ser controlado e mantido entre 2,5-3,0 para que não ocorra a coagulação dos compostos orgânicos.

Sugere-se que para aprimoramento das técnicas, seja realizada a análise do carbono orgânico total após a aplicação dos dois processos para avaliar a eficiência de mineralização dos compostos intermediários possivelmente gerados durante a reação de degradação de fenol. 
Apesar da concentração de fenol remanescente no final dos ensaios por ozonização e Fenton e Foto-Fenton ainda estar acima do limite estipulado pela Resolução CONAMA 430/11, os experimentos realizados demonstraram grande potencial de aplicação das técnicas estudadas na degradação de compostos persistentes e incentivam a aplicação das mesmas para outros contaminantes orgânicos.

\section{REFERÊNCIAS}

APHA-AWWA-WPCF, Standard Methods for the Examination of Water and Wastewater. 19th Edition. American Public Health Association, Washington, DC, 1995.

BABUPONNUSAMI, A.; MUTHUKUMAR, K. Advanced oxidation of phenol: a comparison between Fenton, Electro-Fenton, Sono-electro-Fenton and Photoelectro- Fenton processes. Chemical Engineering Journal, v. 183, p. 1-9, 2012.

JIN, X.; PELDSZUS, S.; HUCK, P.M. Reaction kinetics of selected micropollutants in ozonation and advanced oxidation processes. Water Research, v. 46, p. 6519-6530, 2012.

KARCI,A.; ARSLAN-ALATON, I.; OLMEZ-HANCI, T.; BEKBÖLET, M. Transformation of 2,4-dichlorophenol by H2O2/UV-C, Fenton and photo-Fenton. Journal of Photochemistry and Photobiology A:Chemistry, v. 230, p. 65-73, 2012.

KAVITHA, V.; PALANIVELU K. The Role of Ferrous Ion in Fenton and Photo-Fenton Processes for the Degradation of Phenol. Chemosphere Resourch, v. 55, p.1235-1243, 2004.

KIWI, J.; LOPEZ, A.; NADTOCHENKO, V. Mechanism and kinetics of the OH-radical intervention during Fenton oxidation in the presence of a significant amount of radical scavenger (Cl-). Environmental Science \& Technology, v. 34, p. 2162-2168, 2000.

KUO, W. G. Decolorinzing Dye Wastewater With Fenton's Reagent. Water Research, v. 26, p. 881-886, 1992.

MAHMOUD, A.; FREIRE, R.S. Métodos Emergentes para Aumentar a Eficiência do Ozônio no Tratamento de Águas Contaminadas. Química Nova, v. 30, p. 198-205, 2007.

MATHESWARAN, M.; MOON, S. Influence parameters in the ozonation of phenol wastewatertreatment using bubble column reactor under continuous circulation. Journal of Industrial and Engineering Chemistry, v. 15, p. 287-292, 2009.

RESOLUÇÃO CONAMA No 430/2011 - "Dispõe sobre condições e padrões de lançamento de efluentes" - Publicação DOU no 92, de 16/05/2011, pág. 89.

SOUZA, S.A.G.U; SOUZA, F.B.; SOUZA, A.A.U. Application of individual and simultaneous ozonation and adsorption processes in batch and fixed-bed reactors for phenol removal. Ozone Science Eng., v. 34, p. 259-268, 2012.

STASINAKIS, A. S. Use of Selected Advanced Oxidation Processes (AOPs) for Wastewater Treatment - a mini review. Global NEST Journal, v. 10, p. 376-385, 2008. 
TEIXEIRA, C. P. A. B.; JARDIM, W. F. Estudos comparativos entre diferentes POAs na oxidação de fenol. $21^{\circ}$ Congresso Brasileiro de Engenharia Sanitária e Ambiental, n 47, 2007.

TURHAN, K.; UZMAN, S. Removal of phenol from water using ozone. Desalination, v. 229, p. 257-263, 2008.

WANG, X.; ZENG, G.; ZHU, J. Treatment of jean-wash wastewater by combined coagulation, hydrolysis/acidification and Fenton oxidation. Journal of Hazardous Materials. v. 153 , p. $810-816,2008$.

ZENG, Z.; ZOU, H.; LI, X.; AROWO, M.; SUN, B.; CHEN, J.; CHU, G.; SHAO, L. Degradation of phenol by ozone in the presence of Fenton reagent in a rotating packed bed. Chemical Engineering Journal, v. 229, p. 404-411, 2013.

ZHONG, W.; WANG, D.; XU, X. Phenol removal efficiencies of sewage treatment processes and ecological risks associated with phenols in effluents. Journal of Hazardous Materials. v. 217-218, p. 286-292, 2012. 